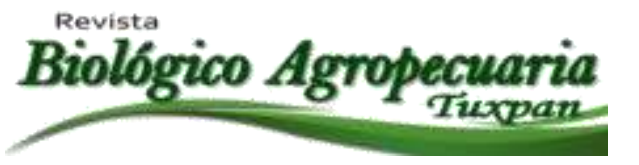

\title{
Crecimiento de dos variedades de mojarra tilapia en jaulas flotantes, bajo tres densidades de
}

\section{población}

Two varieties of tilapia fish growth in floating cages under three population densities

Guadalupe Castillo-Capitán $^{1,6 \bowtie}$, Luis Carlos Alvarado-Gómez ${ }^{2}$, María Gisela Velázquez-Silvestre ${ }^{3}$, Alejandro Retureta-Aponte ${ }^{4}$ y Obet Torres-Alonso ${ }^{5}$

1,2,3,4,5 Facultad de Ingeniería en Sistemas de Producción Agropecuaria, Universidad Veracruzana. Km 4.5 km.- carretera Acayucan - Catemaco, Acayucan, Veracruz México. Tel y Fax (924) 24-791-22

${ }^{\square}$ Autor para la correspondencia: gcastillo@uv.mx

Recibido: $18 / 01 / 2014$
Aceptado: 22/07/2014

\section{RESUMEN}

Se estudió el efecto de diferentes densidades de población sobre el crecimiento de dos variedades de mojarra tilapia, Oreochromis niloticus (variedad Stirling) y Oreochromis ssp (pargo UNAM). El trabajo se realizó bajo el sistema de jaulas flotantes con capacidad de $1 \mathrm{~m}^{3}(3 \times 1 \times 1)$, bajo tres densidades experimentales: 100, 200 y 300 peces $/ \mathrm{m}^{3}$ /Jaula. El experimento se evaluó durante tres meses (marzo, abril y mayo), los peces se alimentaron a libre acceso tomando en cuenta su biomasa corporal, duplicando la alimentación para la densidad de 200 y triplicándola para la densidad de 300 . El crecimiento se evaluó con base en talla y peso, con diseño factorial completamente al azar y diez repeticiones por cada tratamiento, utilizando un ANOVA para el análisis de los datos. Los resultados del experimento nos indican que estadísticamente hay diferencia significativa para ambas variedades. La variedad Stirling tiene un crecimiento significativo y mejor adaptación a las diferentes densidades a la que fue sometida, Pargo-UNAM sólo se adapta y tiene mejor crecimiento con densidad de 100 peces $/ \mathrm{m}^{3}$. Los resultados obtenidos indican que para tener resultados satisfactorios en la producción de tilapia en jaulas flotantes, se deben tomar en cuenta las densidades poblacionales, que son parte funcional de un sistema de producción.

Palabras clave: Oreochromis spp, densidad, jaulas flotantes, crecimiento, biomasa. 


\begin{abstract}
The effect of different population densities on the growth of two varieties of tilapia crappie was studied the first variety was Stirling (Oreochromis niloticus) and the second Pargo-UNAM (Oreochromis spp). The work was performed under the floating cage system with capacity of $1 \mathrm{~m} 3(3 \times 1 \times 1)$ under three experimental densities: 100, 200 and 300 fish/m3/cage. The experiment was evaluated for 3 months (March, April and May), the fish were fed ad libitum considering doubling its body biomass for power density of 200 and tripling the density of 300. Growth was evaluated based on size and weight, using a completely randomized factorial design, with 10 replications per treatment. ANOVA was used for analysis of data Results shown a significant difference for both varieties. Individuals of variety Stirling has significant growth and a better adaptation to all different densities, compared with the PargoUNAM, which only adapts and has better growth in $100 \mathrm{fish} / \mathrm{m}^{3}$ density. However, for optimal performance of Nile tilapia in floating cages, parameters must be considering as they are functional part of a production system.
\end{abstract}

Keywords: Oreochromis spp, density, floating cages, growth, biomass.

\section{INTRODUCCIÓN}

Las tilapias son el grupo de peces con mayor futuro económico en cultivos comerciales y para programas de subsistencia alimentaria, en virtud a sus cualidades de rusticidad y rápido crecimiento; por sus hábitos alimenticios, ya que son omnívoros con buena conversión alimenticia, tienen gran resistencia física, sobre todo a enfermedades, elevada productividad, amplia tolerancia a desarrollarse en condiciones de alta densidad, habilidad para sobrevivir a bajas concentraciones de oxígeno en aguas salinas, además de su capacidad de nutrirse a partir de una gran variedad de alimentos naturales y artificiales (Fitzsimmons, 2000). En México, debido a sus características tropicales, la adaptación de la tilapia ha sido un éxito, lo que hace que sea uno de los principales productores de tilapia en Latinoamérica, pero sus exportaciones son mínimas (Ruiz-Velazco et al. 2006), por lo tanto, se requiere una producción sostenida y organizada que permita lograr la eficiencia del sistema. La producción de mojarra tilapia en jaulas y estanques ha sido evaluada en diversos estudios (Bailey et al. 2000; Cruz y Ridha, 1991; Ouattara et al. 2003;
Ridha, 2006), quienes mencionan que la resistencia, tolerancia y el estrés son factores a considerar dentro de los sistemas de producción de tilapia.

El cultivo de peces en jaulas, a altas densidades (200 a 500 peces o $200 \mathrm{~kg} / \mathrm{m}^{3}$ ) podría convertirse en el medio de expansión más importante y simple en la producción de tilapia (Zetina et al. 2006). En el presente trabajo se evaluó el crecimiento en jaula de dos variedades de tilapia, bajo diferentes densidades de población, lo cual permitió garantizar el proceso productivo del sistema y a su vez, determinar la densidad poblacional de mayor eficiencia en el sistema.

\section{MATERIALES Y MÉTODOS}

La investigación se realizó en la comunidad "Los Cerritos", Municipio de Chinameca, Veracruz, México, ubicada en las coordenadas geográficas $18^{\circ} 04^{\prime} 47^{\prime \prime}$ Latitud Norte y los $94^{\circ} 36^{\prime} 56^{\prime \prime}$ Longitud Oeste, a un altura de $27 \mathrm{msnm}$. El clima de esta región es cálido- húmedo con una temperatura promedio

\title{
Revista Científica Biológico Agropecuaria Tuxpan 2 (1) ISSN: 2007-6940
}


de $26^{\circ} \mathrm{C}$ y una precipitación pluvial de 2500 $3000 \mathrm{~mm}$ (INEGI, 2010).

Se construyeron seis jaulas, con una capacidad de $3 \mathrm{~m}^{3}$ cada una, elaboradas con material de PVC y tela mosquitero. Las jaulas se ubicaron dentro de una represa con dimensiones de $32 \mathrm{~m}$ X $15 \mathrm{~m}$ X $2 \mathrm{~m}$. Se estudió el comportamiento de dos variedades de mojarra: la variedad Stirling (Oreochromis niloticus) y la Variedad Pargo-UNAM (Oreochromis ssp). Se utilizó un arreglo factorial (A $\mathrm{X}$ B) bajo un diseño completamente al azar con 10 repeticiones, donde el factor A representó a las variedades, Stirling y Pargo-UNAM, y el factor B las diferentes densidades de población $(100,200$ y 300 alevines $/ \mathrm{m}^{3}$ ).

Las variables talla y peso, fueron evaluadas por medio de muestreos cada 30 días. Para la determinación de la talla, se utilizó una regla graduada de $30 \mathrm{~cm}$ y para el peso, una balanza granataria digital con capacidad de 200 g. Para el análisis estadístico se utilizó la técnica del análisis de varianza y posteriormente una prueba de medias por el método Tukey (0.05). Con el paquete de Diseños Experimentales de la UANL.

\section{RESULTADOS Y DISCUSIÓN}

Los resultados del análisis de varianza, para la variable peso, presentaron diferencias significativas, entre los genotipos evaluados. El híbrido Pargo-UNAM, superó a la variedad Stirling, con incrementos de $47 \%, 11 \%$ y $20 \%$, para los meses de Marzo, Abril y Mayo, respectivamente. La interacción para la variable peso y densidad, durante el mes de marzo y abril, tuvo mejor combinación en la variedad Pargo -UNAM, con la densidad de 100 peces $/ \mathrm{m}^{3}$. Esto debido a que los híbridos tienen un mayor potencial genético, exhibiendo una mayor plasticidad genética, que se expresa en mayores rendimientos en ambientes favorables (Fig. 1 y 2). Aunque podemos decir que para el caso de la variedad Stirling la mejor combinación sería la de 200 peces $/ \mathrm{m}^{3}$. Esto coincide con Schmittou (1994), quien menciona que cuando los peces son confinados a altas densidades, el espacio individual o colectivo se puede convertir en un factor limitante de la producción.

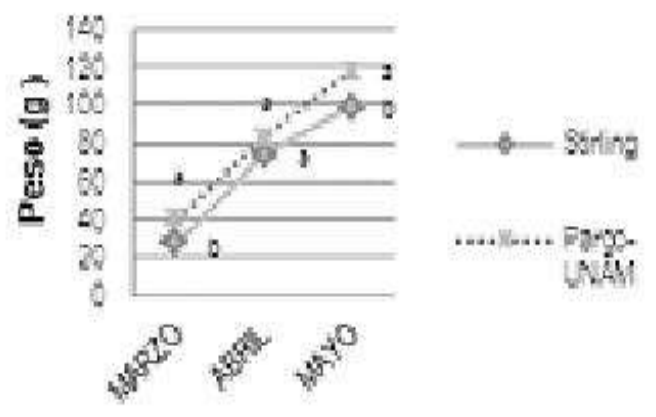

Figura 1. Comportamiento de la variable peso en las dos variedades. 


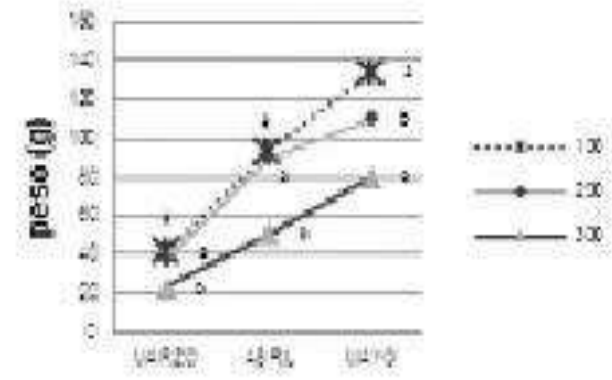

Figura 2. Comportamiento de la variable peso en las tres variedades.

La variedad que mejor talla obtuvo durante todo el experimento fue la PargoUNAM (Fig. 3). Para las diferentes densidades a la que fueron sometidas las variedades, podemos decir que la mejor talla se registró en la densidad de 100 individuos $/ \mathrm{m}^{3}$ (Fig. 4).

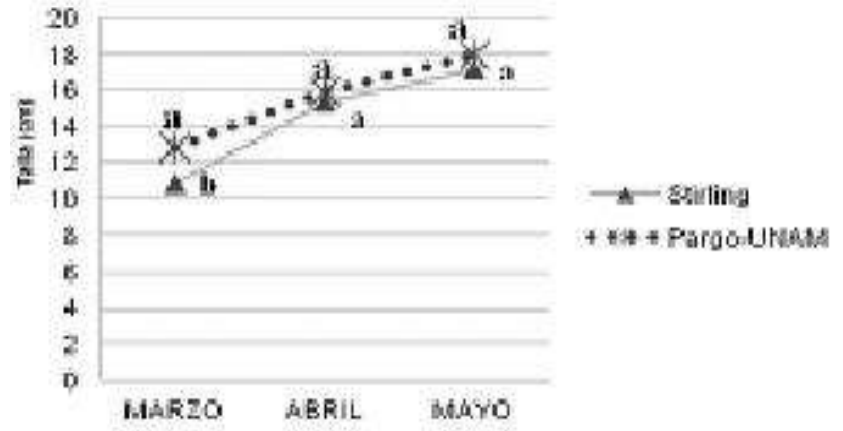

Figura 3. Comportamiento de la variable talla en las dos variedades.

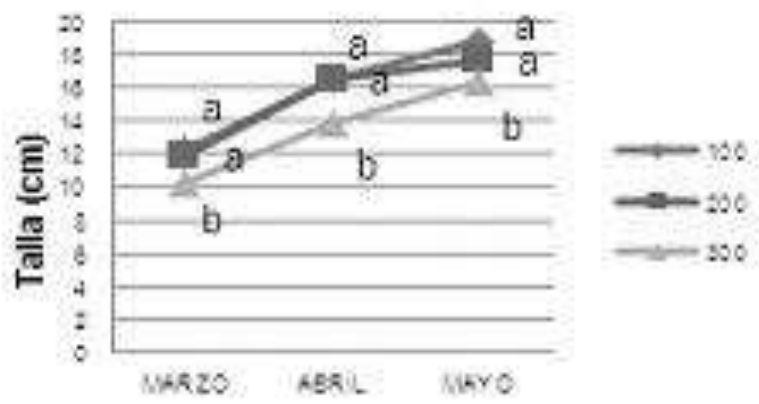


Figura 4. Comportamiento de la variable talla en las tres variedades.

Con respecto a la variable talla, durante el periodo evaluado, la interacción del análisis de varianza, nos indica que la mejor combinación entre variedad y densidad es la Pargo-UNAM con 100 peces $/ \mathrm{m}^{3}$ (Fig. 5). Según Joblin (1993) una alta densidad, además de afectar negativamente la calidad del agua causa la pérdida del apetito de los peces, este es un factor importante que se pudo observar en las jaulas con densidades de 200 y 300. Estos resultados superan a lo dicho por Hardy, 1989; Cruz y Ridha, 1991, quienes consideraron que los peces mantenidos a una densidad de 28 peces $/ \mathrm{m}^{3}$, estuvieron sometidos a un estrés fisiológico mayor que aquellos cultivados a una densidad menor de 14 peces $/ \mathrm{m}^{3}$, lo cual pudiera afectar la capacidad digestiva de los peces.

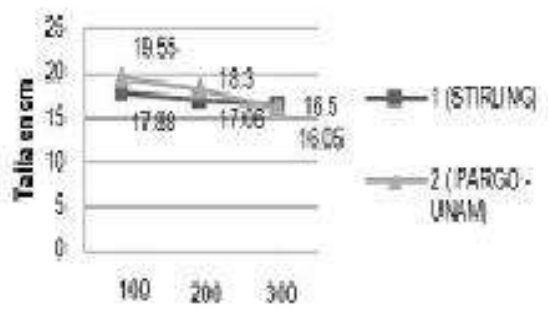

Figura 5. Interacción de las variables talla y densidad.

\section{CONCLUSIÓN}

La densidad de cultivo de mojarra tilapia a diferentes densidades de población, confinados en jaulas flotantes tiene un importante efecto sobre el crecimiento de las variedades Stirling y Pargo-UNAM, en nuestro caso, los mejores resultaron del experimento se obtuvieron cuando los peces se mantuvieron a la densidad de 100 y 200 peces $/ \mathrm{m}^{3}$.

De las dos variedad fue la Variedad Pargo-UNAM, la que mostró mejor comportamiento en la densidad de 100 peces $/ \mathrm{m} 3$.

La variedad Stirling se adapta bien al cultivo en jaulas flotantes y a altas densidades $(100,200$ y 300 peces/m3), a que mantiene su peso y talla, las altas densidades no afectan a la variedad, aunque deben de tomarse en cuenta los parámetros fisicoquímicos del agua, ya que forman parte fundamental para una buena producción.

Para la variable talla, se observó interacción significativa entre genotipo y densidad. Al final de la evaluación, la variedad Stirling presentó una mayor plasticidad fenótipica, ya que se adaptó a diferentes densidades sin afectar su talla. Mientras que el híbrido Pargo-UNAM la talla disminuye en la densidad más alta.

\section{LITERATURA CITADA}

Bailey, D., SRakocy, J. E., Martin, J. M. y Shultz, R. C. 2000. Intensive production of tilapia fingerlings recirculating system, 
In: Fitzsimmons K. and J.C. Filho (eds). Proceeding of the Fifth International Symposium on Tilapia in Aquaculture. Rio de Janeiro, Brazil. pp. 28-333.

Beltrán-Álvarez, R., Sánchez, P. J., Valdez, G. L. y Salas, A. O. 2009. Edad y crecimiento de la mojarra Oreochromis aureus (Pisces: Cichlidae) en la Presa Sanalona, Sinaloa, México. Rev. Biol. Trop., 58: 325-338. https://doi.org/10.15517/rbt.v58i1.5213

Contreras-Sanchez, W. y Fitzsimmons, K. 2006. Tilapia, Sustainable Aquaculture from the New Millennium - Proceedings of the Seventh International Symposium on Tilapia in Aquaculture. American Tilapia Association, Aquaculture CRSP. 389 p. https://doi.org/10.1079/9781845934941.0374

Cruz, E. M. y Ridha, M. T. 1991. Production of the tilapia Oreochromis spilurus Günter stocked at different densities in sea cages. Aquaculture, 99: 95-103. https://doi.org/10.1016/0044-8486(91)90290-n

Fitzsimmons, K. y Carvalho, J. 2000. Tilapia Aquaculture en el siglo 21: Actas del V Simposio Internacional de Acuicultura de Tilapia. Editores: Ministerio de Agricultura, Brasil y Acuicultura CRSP. Río de Janeiro. 682 p.

Granado, A. 1995. Crecimiento del morocoto Piaractus brachypomus (Osteichthyes, Characiformes) en jaulas flotantes. Revista Latinoamericana de Acuicultura, 44: 81-88.

Hardy, R. W. 1989. Diet preparation. En J.E. Halver Ediciones, Fish Nutrition 2da Edición, Academic Press, USA. pp. 475548.

INEGI, 2010. Censo de Población y vivienda 2010. Veracruz. Instituto Nacional de
Estadística, Geografía e Informática. México.

En:http://www.inegi.org.mx/sistemas/con sulta_resultados/iter2010.aspx?c $=27329 \&$ s=est (consultado el 28 de septiembre de 2012). https://doi.org/10.17013/risti.26.43-53

Joblin, M. 1993. Nutrition, diet fromulation and feeding practices. En K. Heen, R. L. Monahan y F. Uther, Editores. Salmon Aquaculture, Fishing News Books, USA. pp. 83-126.

Khallaf, E. A., Galal, M. y Authman, M. 2003. The biology of Oreochromis niloticus in a polluted canal. Ecotoxicol, 12: 405-416. https://doi.org/10.1023/a:1026156222685

Ridha, M. T. 2006. Comparative study of growth performance of three strains of Nile tilapia, Oreochromis niloticus, L. at two stocking densities. Aquaculture Research, 37: 172-179. https://doi.org/10.1111/j.1365-2109.2005.01415.x

Ruiz, V., Tapia, A. J., García, J. P. y González, V. H. 2006. Evaluación de un cultivo semi-Intensivo de tilapia (Oreochromis niloticus) en tanques circulares con aguas termales. Revista Electrónica de Veterinaria REDVET, 7(11): 1- 22.

Schmittou, H. R. 1994. Cultivo de peces a alta densidad en jaulas de bajo volumen. Asociación Americana de Soya. 86 p.

Zetina, C. P., Reta M .J. L., Olguín, P. C., Acosta, B. R. y Espinosa, S. G. 2006. El cultivo de Tilapia (Oreochromis spp.) en la rentabilidad de seis agroecosistemas de Veracruz. Técnica Pecuaria, México, 44 (2): 169-179.

\section{Revista Científica Biológico Agropecuaria Tuxpan 2 (1) ISSN: 2007-6940}


Copyright (c) 2014 Guadalupe Castillo Capitán, Lvis Carlos Alvarado Gómez, Maria Gisela Velázxuez Silvestre, Alejandro Retureta Aponte yObed Torres Alonso

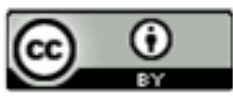

Este texto está protegido por una licencia Creative Commons 4.0

Usted es libre para Compartir —copiar y redistribuir el material en cualquier medio o formato- y Ad aptar el documento —remezclar, transformar y crear a partir del material- para cualquier propósito, incluso para fines comerciales, siempre que cumpla la condición de:

Atribución Usted debe dar crédito a la obra original de manera adecuada, proporcionar un enlace a la licencia, e indicar si se han realizado cambios. Puede hacerlo en cualquier forma razonable, pero no de forma tal que sugiera que tiene el apoyo del licenciante olo recibe por el usoque hace de la obra.

$\underline{\text { Resumendelicencia - Textocampletodklalicerria }}$

Revista Científica Biológico Agropecuaria Tuxpan 2 (1)

ISSN: 2007-6940 